\title{
Development of a low-cost reliable indoor localization system targeted to older users with mild cognitive impairments
}

\author{
Carlos A Velasco, Yehya Mohamad and Alan Koshy John \\ Fraunhofer Institute for Applied Information Technology FIT \\ Schloss Birlinghoven, D53757 Sankt Augustin (Germany) \\ URL: http://www.fit.fraunhofer.de/
}

\begin{abstract}
The "Web of Things is about extending the Internet of Things [Ashton, 2009] concept beyond the connection of things and considering issues like heterogeneity, scalability and usability with respect to progressive computing” [Mayer et al., 2013]. By introducing web technologies, the concept overpasses Machineto-Machine (M2M) communication and incorporates interaction with users into the landscape. This paper presents a technical overview of our preliminary work developing a low-cost and reliable indoor localization system using state-of-the-art Web of Things technologies targeted to support the daily activities of users with mild cognitive impairments and their carers. This work extends previous experiences of the authors using technologies like RFID [Gappa et al., 2012].

Index Terms-indoor positioning system, Bluetooth Low Energy (BLE), wearable computing and innovative mobile devices, propagation model, Kalman filter, persons with special needs, mild cognitive impairments.
\end{abstract}

\section{INTRODUCTION}

The seminal papers from Weiser [1], and Ishii [2] presented a new paradigm of Human-Computer Interaction where the interfaces surround the user embedded in everyday artifacts. These pioneering works became mainstream at the end of the previous decade with the arrival of smart phones and the so-called Internet of Things [3], extended to the Web of Things (see, e.g., [4] and references therein), enabled by architectural principles based upon the Representational State Transfer (REST, [5], [6]).

These works and many that followed them presented a variety of multimodal user interfaces, which allowed the user to interact with her environment and exchange information with others. However, very few of this research has addressed the requirements of people with special needs (elderly and people with disabilities) for these interfaces. These requirements have a growing importance bearing in mind the population aging in Europe [7] and worldwide, and that these user groups need access to critical services such as health networks (e.g., patient adherence), transport, communications (e.g., social networks) or smart environments (Ambient Assistive Living, AAL), etc.

Our target user group is affected by a decline in physical and cognitive abilities, which reduces their opportunities to live independently. Therefore, there is a high demand on the provision of IT solutions that prolong the independent living at home of this user group [8]. The Web of Things is the most suitable technology to provide support to this user group, combined with the use of different assistive devices. Under the scope of our WebDA ${ }^{1}$ (Web-based Services for Elderly People and their Caregivers) project, we have investigated different technologies that allowed as the implementation of different support services like a localization service, an action planner and several other scaffolding services.

The localization service developed within the WebDA project was based upon RFID technologies. A thorough user testing of the developed prototypes in real environments showed a lot of limitations from a reliability perspective, maintenance and installation costs, and overall user-friendliness [9].

Since the end of our project, there were many advances in sensor technologies that could improve the quality of our approach. Although in WebDA we have addressed indoor localization of objects and persons, we have investigated the use of Bluetooth Low Energy (BLE [10]) systems because of their low cost and simplicity of installation.

The rest of the paper is organised as follows: section II presents a brief discussion of the state-of-the-art on indoor localization technologies; section III] introduces a preliminary set of user and system requirements; section [V] presents a technical overview with the architecture of our system, including some discussion on implementation decisions; and finally, section $\mathrm{V}$ presents our preliminary conclusions.

\section{STATE-OF-THE-ART OF INDOOR LOCALIZATION SERVICES}

Whilst outdoor localization is technically a well solved problem through the Global Positioning System (GPS) or its European equivalent GALILEO, indoor localization still remains an open issue for academia and industry [11]. The GPS signals from satellites are attenuated and scattered by roofs, walls and other objects. The approaches towards solving the indoor localization problem can be classified into infrastructure-free and infrastructure-based approaches.

Infrastructure-free approaches focus on leveraging already existing wireless signals, such as the earth's geomagnetic field strength. In [12], the internal geomagnetic map of a building is analyzed and its variation was measured. A mobile phone was

${ }^{1}$ http://webda.info/ (available only in German) 
used to detect the field and based on the pre-determined map the exact location was obtained. This technology is becoming a niche for many start-ups such as IndoorAtlas ${ }^{2}$ or Micello 3

Infrastructure-based approaches rely on the deployment of customized RF-beacons. For example, a Microsoft Research group developed RADAR [13], the first WiFi-based system for locating and tracking users inside buildings. RADAR operates by recording and processing WiFi signal strength information at multiple base stations positioned to provide overlapping coverage in the area of interest. It employs techniques that combine empirical measurements with signal propagation modeling to enable location-aware services and applications

A system based on infrared (IR) technology is described in [14]. IR transmitters are attached at known positions, usually ceilings, in a building. An optical sensor senses the IR beacons, which enables the system software to determine the user's location. IR-systems suffer from several drawbacks: (i) they perform poorly due to their limited range, (ii) the presence of direct sunlight in rooms affects their performance, and (iii) it incurs in significant installation and maintenance costs.

Another technology of interest is Radio-frequency identification (RFID), which uses electromagnetic fields to transfer data, for the purposes of automatically identifying and tracking tags attached to objects [15]. This approach requires a number of RFID readers to be installed. Each reader has a predetermined power level, hence only a certain unique region is covered by this reader. The whole indoor location can be thus divided into smaller regions by placing adequate number of readers. Given an RFID tag, one can associate that tag with a known sub-region. The accuracy of this approach is determined by the number of readers required, hence it is very expensive and more number of readers may cause interferences. This technology is successfully used in the logistics industry, where large investments are justified on account of efficiency and location efforts, but are hardly justifiable in the context of Ambient Assisted Living (AAL), as demonstrated in our WebDA research project [9], [16].

\section{USER AND SYSTEM REQUIREMENTS}

After the experiences gather in our WebDA project, we investigated how could we improve the person localization by using other technologies different from RFID. With that, we could try to implement our scaffolding context-aware services ([17], [18]) with an affordable infrastructure. Due to this we have used emerging beacon technologies based upon Bluetooth Smart [10], [19].

Our system has 2 types of actors:

(i) the end user, who needs a very simple interface based upon context-aware reminders (provided in the first prototype via audio messages and vibration alerts), which depend on time and location; and

(ii) the caregiver (who can be a family member or a professional), who needs an additional interface to

${ }^{2}$ https://www.indooratlas.com/features

3 http://www.micello.com/ prepare customized messages and may need to monitor special situations such as restlessness at night, leaving the house and wandering outside the house.

The use cases implemented in the first prototype are tailored to these two actors. Caregivers could access via a web application our action planner [17], to create customized contextaware messages and actions via our controlled natural language service. And end users will receive via a mobile device these reminders as voice messages or vibration notifications (for example, reminders to take a medicine at given times, to drink water when the user is in the kitchen, to stand up after a long inactivity periods in the living room, etc). Our development environment has a restriction that we did not have in WebDA, because in our first prototypes we did not aim to locate objects.

\section{Architectural OVERVIEW OF THE System}

The architecture of our system (see Fig. 1) is composed of two main systems, which are described in more detail in the following subsections:

(i) server-side components that manipulate complex and process intensive modules, like those related to the persistence layer, security, algorithms processing, etc.; and

(ii) client-side components that are presented to the 2 types of users described above. This client-side access is happening in our system through mobile apps or through web applications, depending on the different use cases.

\section{A. Server-side components}

Our server prototype has the following main components:

(i) Filtering modules: these are modules that handle sensor signals, such as reduction filters, trilateration calculations, etc. (see section IV-C for more details).

(ii) Sensor management module: this module handles and calibrates the different sensors of the system (either integrated into the mobile device like the accelerometer or the magnetometer, for instance, or external infrastructure sensors like the BLE beacons). This module also takes care of the data persistence, metadata storage, etc.

(iii) User Interface (UI) modules: responsible for the management of the interfaces of the client applications.

(iv) Generic controller module: responsible of the manipulation of the auxiliary services and business logic of the applications, such as security, event logging, user administration and access control, etc.

The persistence layer of the application is implemented via a NoSQL back-end that ensures a dynamic access to the big amount of data generated by the system.

\section{B. Client-side components}

Client-side components are divided in two types of applications depending on the target user group as described in 


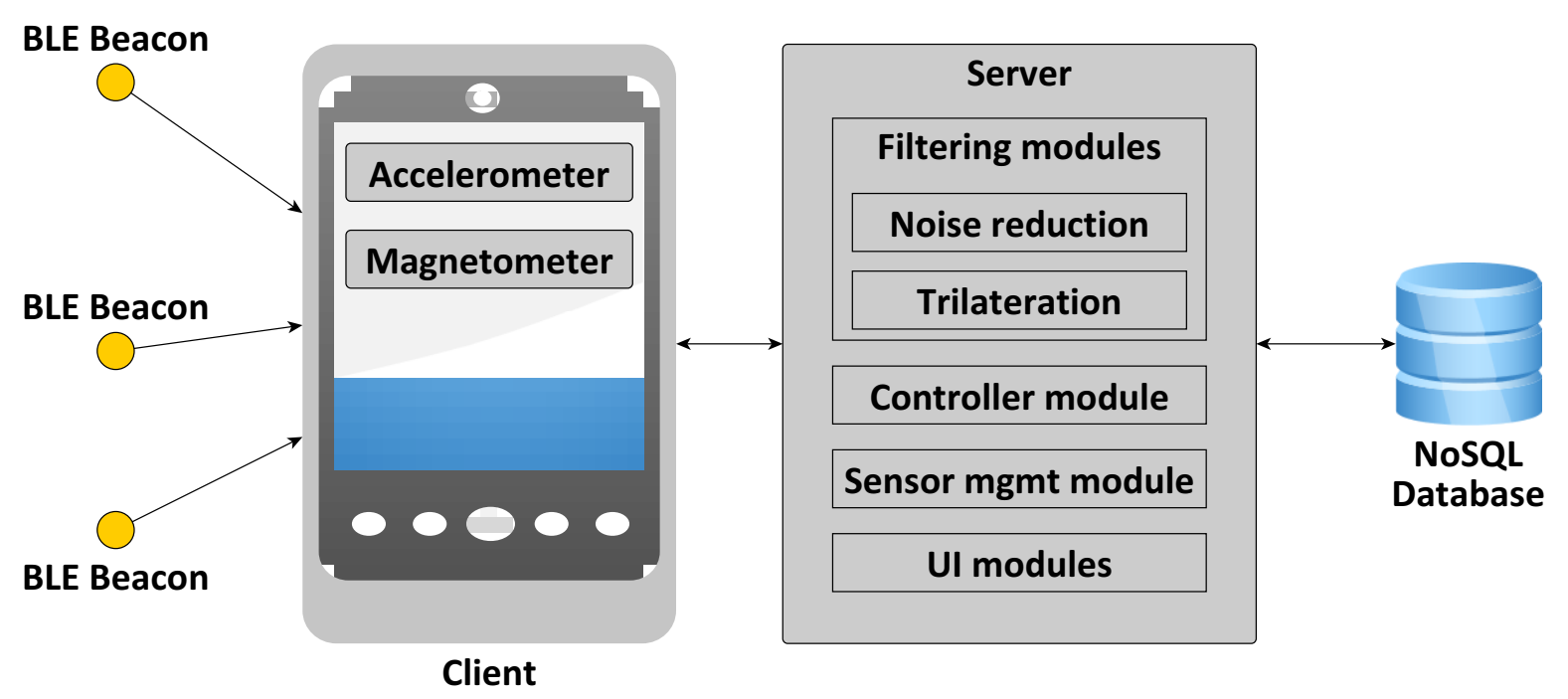

Fig. 1. Schematic architectural overview of the indoor localization system.

section III. There is an additional interface for the administration of the system and the configuration of the different components.

Caregivers have access to a web application that allows them to customize the messages and alerts of the system for the end users. It should also allow the possibility to record and store voice messages.

End users have access via a preconfigured mobile app to these context-aware alerts. In our first prototype, they are implemented via the vibration of the device and by playing a pre-recorded audio message of the caregiver.

\section{Location algorithms}

The geometrical problem of locating a person or an object through three or more known distances to reference points is a trivial mathematical endeavor [20]. However, when dealing with electronic means to measure distances, there is a degree of uncertainty due to valuations introduced by the environment on those means. Then, we meet with an ill-conditioned positioning problem that can be solved in different ways (for instance, [21] addresses the solution comparing different least square approximation methods).

Additionally, the mechanisms that govern radio propagation are very complex, as they are afflicted by reflection, diffraction and scattering of radio waves (see [22] and references therein). Therefore, the spherical model as a propagation model must be quickly rejected.

For our prototype implementation, we have chosen the model for indoor propagation described in [23]. The model is based upon the Received Signal Strength Indication (RSSI), which is one of the signal-related parameters made available by Bluetooth specification. RSSI is an 8-bit signed integer that denotes whether the received power level is above or below the Golden Receive Power Range (GRPR), which is considered the optimal value. A positive or negative RSSI (in
$\mathrm{dB}$ ) means the received power level is above or below the GRPR, respectively, while a zero implies that it is ideal. The RSSI value reported by the beacon can be used to measure the distances between the beacon and the receiving mobile device [24].

$$
R S S I=-10 n \log _{10} d+A
$$

where the Radio Frequency (RF) parameters $A$ and $n$ are used to describe the network environment. The RF parameter $A$ is defined as the absolute energy (in $\mathrm{dBm}$ units) at a distance of $1 \mathrm{~m}$ (meter) from the transmitter, which is the ideal RSSI reading at $1 \mathrm{~m}$ from the transmitter; $n$ is the signal transmission constant and it is relevant to signal transmission environment; and $d$ is the distance from the transmitter node to the receiving node.

To calculate the distance $d$, the environment factor, $n$ has to be calibrated in the test environment. For that, we have calculated the parameter $n$ at different distances measuring for every beacon (i) a calibrated distance and its RSSI. From Eq. (1), this is done via:

$$
n_{i}=-\left(\frac{R S S I_{i}-A}{10 \log _{10} d_{i}}\right)
$$

A simple quadratic curve fitting could to be done to obtain a valid $n$ value for the given environment. The main problem is that for most of the radio frequencies, RSSI varies a lot because of the issues mentioned earlier. Therefore, it is necessary to filter them. Since RSSI values follow a normal distribution model, we have used Chebyshev's outlier detection method. According to Chebyshev's theorem, at least $\left(1-1 / k^{2}\right)$ of the distribution's values are within $k$ standard deviations of the mean. The value of $k$ was chosen as 2 [25]. By using a value of $2,75 \%$ of the values have to lie within two standard deviations of the mean, according to the theorem. RSSI values 
not falling in this range were eliminated. Once the outliers were removed, a Kalman filter is used to get a better estimate of the signal.

The Kalman filter is an efficient recursive filter that statistically estimates the internal state of a system from a series of measures which are subject to noise [26]. A suitable Kalman model is created by using a series of RSSI readings measured in time slots of $3 s$. The covariance matrix of the estimation, $P$, is initialized with the variance of the readings. The value of the update matrix, $F$, is chosen as 1 as we expect that the user carrying the mobile device moves slowly. We assume that the process noise, $Q$, is negligible. The measurement noise, $R$, is calculated from the average RSSI value and using (1).

To avoid interferences between the beacons and to optimize their battery life, we have set up an average range of $10 \mathrm{~m}$ to every beacon and placed them in different rooms. With that model in mind, we were able to reliably detect the presence of a user within a room. Location predictions still show an error which oscillates between 1 and $3 \mathrm{~m}$. At the moment of writing this paper we are trying to improve this location predictions via the integration of other sensor readings, such as those coming out of the accelerometer and magnetometer in the mobile device.

To facilitate the calibration process, we have also developed in the client-side a calibration app that supports the installation of the system in different environments (see Fig. 2).

\section{CONCLUSiOns}

We have presented a brief overview of our approach and the implemented system at the time of writing the paper. Current work is focused on the following points:

(i) improvement of the location accuracy by integrating readings from other sensors available to the system;

(ii) enhancement of the implemented user interfaces targeted to caregivers and end users; and

(iii) preliminary preparations to test the system with real users.

The actual prototype is developed for a mobile device (smart phone or tablet). Further work will be necessary to integrate into the system different type of wearables, such as smartwatches or armbands.

\section{REFERENCES}

[1] M. Weiser, "The computer for the 21st century," Scientific American, vol. 265, no. 3, pp. 66-75, Sep. 1991.

[2] H. Ishii and B. Ullmer, "Tangible bits: Towards seamless interfaces between people, bits and atoms," in Proceedings of the ACM SIGCHI Conference on Human Factors in Computing Systems, ser. CHI '97. New York, NY, USA: ACM, 1997, pp. 234-241. [Online]. Available: http://doi.acm.org/10.1145/258549.258715

[3] K. Ashton, "That "internet of things" thing," RFiD Journal, vol. 22, p. 97-114, 2009

[4] S. Mayer, V. Trifa, D. Raggett, and D. Guinard, "Wot 2013: Fourth international workshop on the web of things," in Proceedings of the 2013 ACM Conference on Pervasive and Ubiquitous Computing Adjunct Publication, ser. UbiComp 13 Adjunct. New York, NY, USA: ACM, 2013, pp. 1487-1494. [Online]. Available: http: //doi.acm.org/10.1145/2494091.2497388

\section{Calibration}

\author{
Tx Power
}

「x Power

$0.5 \mathrm{~m}$

RSSI

$2 m$

RSSI

$3 m$

\section{RSSI}

$5 \mathrm{~m}$

\section{RSSI}

$8 \mathrm{~m}$

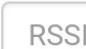

RSSI

$10 \mathrm{~m}$

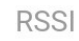

$\triangleleft$

O

$\square$

Fig. 2. User interface of the calibration app of our system.

[5] R. T. Fielding, "Architectural styles and the design of network-based software architectures," Ph.D. dissertation, University of California, Irvine, 2000, aAI9980887.

[6] R. T. Fielding and R. N. Taylor, "Principled design of the modern web architecture," ACM Trans. Internet Technol., vol. 2, no. 2, pp. 115-150, May 2002. [Online]. Available: http://doi.acm.org/10.1145/ 514183.514185

[7] Directorate-General for Economic and Financial Affairs of the European Commission. (2011, 4) The 2012 Ageing Report: Underlying Assumptions and Projection Methodologies. [Online]. Available: http://bit.ly/1dFIg7n

[8] Federal Statistical Office of Germany. (2011) Older people in Germany and the EU. [Online]. Available: http://bit.ly/1dFIrQ8

[9] H. Gappa, G. Nordbrock, J. Pullmann, and Y. Mohamad, "Webbasierte Dienste für ältere Menschen und Angehörige (WebDA) Schlussbericht," Fraunhofer FIT, Tech. Rep., 2013. [Online]. Available: http://bit.ly/1KcI4FV

[10] Bluetooth SIG, Inc. (2015) Bluetooth Smart Technology: Powering the Internet of Things. [Online]. Available: http://www.bluetooth.com/ Pages/Bluetooth-Smart.aspx

[11] D. Lymberopoulos, J. Liu, X. Yang, R. R. Choudhury, V. Handziski, and S. Sen, "A realistic evaluation and comparison of indoor location technologies: Experiences and lessons learned," in Proceedings of the 14th International Conference on Information Processing in Sensor Networks, ser. IPSN '15. New York, NY, USA: ACM, 2015, pp. 178189. [Online]. Available: http://doi.acm.org/10.1145/2737095.2737726

[12] J. Chung, M. Donahoe, C. Schmandt, I.-J. Kim, P. Razavai, and M. Wiseman, "Indoor location sensing using geo-magnetism," in 
Proceedings of the 9th International Conference on Mobile Systems, Applications, and Services. New York, NY, USA: ACM, 2011, pp. 141154. [Online]. Available: http://doi.acm.org/10.1145/1999995.2000010

[13] P. Bahl and V. N. Padmanabhan. (2000) RADAR: an in-building rf-based user location and tracking system. [Online]. Available: http://research.microsoft.com/en-us/groups/sn-res/infocom2000.pdf

[14] R. Azuma, "Tracking requirements for augmented reality," Commun. $A C M$, vol. 36, no. 7, pp. 50-51, Jul. 1993. [Online]. Available: http://doi.acm.org/10.1145/159544.159581

[15] L. M. Ni, Y. Liu, Y. C. Lau, and A. P. Patil, "Landmarc: Indoor location sensing using active rfid," Wireless Networks, vol. 10, no. 6, pp. 701-710, 2004. [Online]. Available: http: //dx.doi.org/10.1023/B\%3AWINE.0000044029.06344.dd

[16] H. Gappa, G. Nordbrock, A. Johannen, and A. Schmitz, "Implications of dementia and age-related memory impairments on the design of it-based systems," in Deutscher AAL-Kongress. VDE Verlag, 2012.

[17] H. Gappa, G. Nordbrock, Y. Mohamad, J. Pullmann, and C. A Velasco, "Controlled natural language sentence building as a model for designing user interfaces for rule editing in assisted living systems - a user study," in Computers Helping People with Special Needs, ser. Lecture Notes in Computer Science, K. Miesenberger, A. Karshmer, P. Penaz, and W. Zagler, Eds. Springer Berlin Heidelberg, 2012, vol. 7383, pp. 415-418. [Online]. Available: http://dx.doi.org/10.1007/978-3-642-31534-3_61

[18] H. Gappa, G. Nordbrock, M. Thelen, J. Pullmann, Y. Mohamad, and C. A. Velasco, "Extended scaffolding by remote collaborative interaction to support people with dementia in independent living - a user study," in Computers Helping People with Special Needs, ser. Lecture Notes in Computer Science, K. Miesenberger, D. Fels, D. Archambault, P. Peňáz, and W. Zagler, Eds., vol. 8547. Springer International Publishing, 2014, pp. 445-450. [Online]. Available: http://dx.doi.org/10.1007/978-3-319-08596-8_69
[19] Estimote, Inc. (2015) Beacon Tech Overview. [Online]. Available: http://developer.estimote.com/

[20] Wikipediaß). (2015) Trilateration. [Online]. Available: https://en. wikipedia.org/wiki/Trilateration

[21] W. S. Murphy and W. Hereman, "Determination of a position in three dimensions using trilateration and approximate distances," 1995.

[22] J. Andersen, T. Rappaport, and S. Yoshida, "Propagation measurements and models for wireless communications channels," Communications Magazine, IEEE, vol. 33, no. 1, pp. 42-49, Jan 1995.

[23] Y. Wang, X. Yang, Y. Zhao, Y. Liu, and L. Cuthbert, "Bluetooth positioning using rssi and triangulation methods," in Consumer Communications and Networking Conference (CCNC), 2013 IEEE, Jan 2013, pp. 837842.

[24] J. Albowicz, A. Chen, and L. Zhang, "Recursive position estimation in sensor networks," in Network Protocols, 2001. Ninth International Conference on, Nov 2001, pp. 35-41.

[25] B. Amidan, T. Ferryman, and S. Cooley, "Data outlier detection using the chebyshev theorem," in Aerospace Conference, 2005 IEEE, March 2005, pp. 3814-3819.

[26] R. E. Kalman, "A new approach to linear filtering and prediction problems," J. Basic Eng., vol. 82, no. 1, pp. 35-45, 1960. 